\title{
Disparity of Macrodispersivity Estimated from Temporal and Spatial Moments Using Random Walk Particle Tracking in Heterogeneous Porous Formations
}

\author{
Kazuya Inoue ${ }^{1}$, Gerard J.M. Uffink ${ }^{2}$, Akira Kobayashi ${ }^{3}$, \\ Naoko Matsunaga ${ }^{4}$ and Tsutomu Tanaka ${ }^{5}$
}

\begin{abstract}
This paper presents advective and dispersive solute transport processes in naturally heterogeneous porous formations based on a random walk particle tracking method. Specifically, the disparity of macrodispersivities estimated from temporal moments of breakthrough curves at control planes and from spatial moments of particle cloud is investigated in non-uniform flow systems under the three different conditions of hydraulic conductivity distributions. For both temporal and spatial moment approaches, longitudinal macrodispersivity estimates in stratum porous formations are in considerable agreement with the results reported in the literature. The disparity of macrodispersivity attributes to the nature of both approaches to evaluate a travel distance of particle plume. While the second central temporal moments in stratum aquifer asymptotically approach a constant value regardless of the injection and pumping rates at active wells, the behavior of the second spatial moments depends upon the porous formations and pumping rates. Furthermore, spatial moment approach represents a potentially valuable tool in the assessment of transverse macrodispersion process in heterogeneous porous media.
\end{abstract}

Keywords: $\quad$ Random walk particle tracking; Macrodispersivity; Spatial moment; Temporal moment; Heterogeneity

\section{Introduction}

Solute transport in porous media is fundamental to many significant geoenvironmental problems. It is now well recognized that natural porous media exhibit spatial variability at the continuum scale and that this variability has a profound impact upon solute fate and transport at the larger field scale relevant to environmental and hydrogeological problems. Efforts to apply dispersive solute transport models under field conditions have demonstrated that field-observed dispersive coefficients can be several orders of magnitude larger than those indicated by laboratory tests with similar materials (e.g., Mackay et al., 1986; LeBlanc et al., 1991). The effect of spatial distribution of hydraulic conductivity on reactive and non-reactive solute transport is enhanced spreading, a phenomenon referred to as macrodispersion (Gelhar and Axness, 1983).

A theory of macrodispersion in aquifers based on a Lagrangian approach has been developed which links spatial and temporal moments and field measurable quantities such as hydraulic conductivity (e.g., Tompson, 1993; Lee et al., 2007). Macrodispersivities are often estimated in the field by

\footnotetext{
${ }^{1}$ Assistant Professor, Graduate School of Agricultural Science, Kobe University, 1-1 Rokkodai-cho, Nada-ku, Kobe 657-8501, Japan (Corresponding Author) E-mail: mornel@kobe-u.ac.jp

${ }^{2}$ Associate Professor, Faculty of Civil Engineering \& Geosciences, Delft University of Technology, Stevinweg 1, Delft 2628CN, The Netherlands

${ }^{3}$ Associate Professor, Graduate School of Agricultural Science, Kyoto University, Kitashirakawa-oiwake-cho, Sakyo-ku, Kyoto 606-8502, Japan

${ }^{4}$ Associate Engineer, Mitsubishi UFJ Information Technology Co. Ltd., 2-7-24 Nihonbashi, Chuo-ku, Tokyo 103-0027, Japan

${ }^{5}$ Professor, Graduate School of Agricultural Science, Kobe University, 1-1 Rokkodai-cho, Nada-ku, Kobe 657-8501, Japan
}

monitoring breakthrough curves at a limited number of observation wells (Freyberg, 1986; Taylor and Howard, 1987). In a Lagrangian framework, temporal moments provide a measure of the non-Fickian behavior of transport in heterogeneous porous media and are therefore of concern. However, field tracer test, in genral, based on breakthrough curves cannot infer transverse dispersivity. In the same manner as the temporal moments, a snapshot of a cloud of solute particles in time expresses the dispersive variation in the hydraulic conductivity field, leading to the estimates of spatial moments corresponding to the heterogeneity (Naff, 1990; Salamon et al., 2006). Gelhar et al. (1992) illustrated the scaledependence of longitudinal dispersivity, although no descriptions related to the difference between dispersivities derived from temporal and spatial moments were given. Relatively few investigators have considered the disparity of macrodispersivities derived from spatial and temporal moments in a field condition where large-scale variability is of importance as well as macrodispersivities under the differences between flow conditions associated with non-uniform flow paths taking the injection and extraction of groundwater into account.

The objective of this paper is to investigate the differences in the scale-dependence macrodispersivity estimates based on temporal and spatial moments in heterogeneous porous formations. The aquifer system investigated here relies on the hydrogeologic data in the southwest of the Netherlands and is modeled a homogeneous aquifer and two different hydraulic conductivity fields consisting of a stratified aquifer and a geostatistically-induced aquifer. Numerical tracer transport experiments using random walk particle tracking are conducted in these aquifers with injection and 
pumping at wells for estimating macrodispersivity in terms of spatial moments of particle positions and temporal moments of particle arrival times at control planes.

\section{Advective-dispersive particle transport}

\subsection{Seepage flow}

For the transport simulations, a saturated velocity field within a heterogeneous porous medium must initially be developed. The governing equation for hydraulic head distribution in a steady-state, three-dimensional aquifer system with recharge and discharge is usually written as (Bear, 1972)

$$
\frac{\partial}{\partial x_{i}}\left(K(\boldsymbol{x}) \frac{\partial h}{\partial x_{i}}\right)=Q(\boldsymbol{x})
$$

where $x_{i}$ are the Cartesian coordinates, $K(\boldsymbol{x})$ is the hydraulic conductivity at vector location $x, h$ is the hydraulic head at vector location $\boldsymbol{x}, Q(\boldsymbol{x})$ is the steady-state recharge and discharge at vector location $x$. Summation over repeated indices is assumed. The local medium fluid velocity can be determined from

$$
v_{i}(\boldsymbol{x})=-\frac{K(\boldsymbol{x})}{n_{p}} \frac{\partial h(\boldsymbol{x})}{\partial x_{i}}
$$

where $v_{i}(\boldsymbol{x})$ is the pore velocity at vector location $\boldsymbol{x}$ and $n_{p}$ is the porosity.

\subsection{Random walk particle tracking}

Random walk particle tracking method is commonly used in the field of statistical physics to model and analyze processes involving diffusion. In the hydrologic community, the approach has been widely applied to simulate advective and dispersive (diffusive) mass transport problems in subsurface system (e.g., Tompson and Gelhar, 1990; Uffink, 1983; Inoue et al., 2007).

Simulation of advective and dispersive mass transport may proceed by changing particle positions with time via an ItôTaylor integral scheme, given as (Gardiner, 2004)

$$
\boldsymbol{X}_{p}(t+\Delta t)=\boldsymbol{X}_{p}(t)+\boldsymbol{A}\left(\boldsymbol{X}_{p}, t\right) \Delta t+\boldsymbol{B}\left(\boldsymbol{X}_{p}, t\right) \boldsymbol{\Xi}(t) \sqrt{\Delta t}
$$

where $\boldsymbol{X}_{p}(t)$ is the $i$-component of the particle location at time $t, \Delta t$ is the time increment, and $\boldsymbol{\Xi}$ is a vector which contains three normally distributed random numbers with zero mean and unit variance. The vector $\boldsymbol{A}$ represents the deterministic drift determined by the fluid flow velocity and contains contributions from the dispersion tensor. The matrix $\boldsymbol{B}$ represents the direction displacement distance for the random process $\Xi(t) \sqrt{\Delta t}$, which expresses Brownian motion.

Itô (1951) demonstrated that the particle density distribution $f\left(\boldsymbol{X}_{p}, t\right)$, which is defined as the probability of finding a particle within a given interval between $\boldsymbol{X}_{p}$ and $\boldsymbol{X}_{p}+d \boldsymbol{X}_{p}$ at a certain time $t$, fulfills in the limit of large particle numbers and infinitesimally small step size, the so-called FokkerPlanck equation. This equation describing the motion of the particle density distribution $f$ is compared with the advection and dispersion equation and is given by the summation form (Uffink, 1990)

$$
\frac{\partial f}{\partial t}=-\nabla \cdot(\boldsymbol{A} f)+\frac{1}{2} \nabla \nabla:\left(\boldsymbol{B} \cdot \boldsymbol{B}^{T} f\right)
$$

In order to establish an analogy between the familiar advection and dispersion and the Fokker-Planck equations, the former can be expressed as follows (Zheng and Bennett, 1995):

$$
\frac{\partial\left(n_{p} c\right)}{\partial t}=-\nabla \cdot\left(\boldsymbol{v} n_{p} c\right)+\nabla \cdot\left(n_{p} \boldsymbol{D} \cdot \nabla c\right)
$$

where $c$ is the dissolved concentration of solute, $v$ is the pore velocity and $D$ is a velocity-dependent dispersion tensor with the effective molecular diffusion given by as follows (Burnett and Frind, 1987a; Zheng and Bennett, 1995).

$$
\begin{aligned}
& D_{11}=\alpha_{L} \frac{v_{1}^{2}}{v}+\alpha_{T H} \frac{v_{2}^{2}}{|\boldsymbol{v}|}+\alpha_{T V} \frac{v_{3}^{2}}{|\boldsymbol{v}|}+D_{d} \\
& D_{22}=\alpha_{T H} \frac{v_{1}^{2}}{|\boldsymbol{v}|}+\alpha_{L} \frac{v_{2}^{2}}{|\boldsymbol{v}|}+\alpha_{T V} \frac{v_{3}^{2}}{|\boldsymbol{v}|}+D_{d} \\
& D_{33}=\alpha_{T V} \frac{v_{1}^{2}+v_{2}^{2}}{|\boldsymbol{v}|}+\alpha_{L} \frac{v_{3}^{2}}{|\boldsymbol{v}|}+D_{d} \\
& D_{12}=D_{21}=\left(\alpha_{L}-\alpha_{T H}\right) \frac{v_{1} v_{2}}{|\boldsymbol{v}|}+D_{d} \\
& D_{13}=D_{31}=\left(\alpha_{L}-\alpha_{T V}\right) \frac{v_{1} v_{3}}{|\boldsymbol{v}|}+D_{d} \\
& D_{23}=D_{32}=\left(\alpha_{L}-\alpha_{T V}\right) \frac{v_{2} v_{3}}{|\boldsymbol{v}|}+D_{d}
\end{aligned}
$$

where $\alpha_{L}$ is the longitudinal dispersivity, $\alpha_{T H}$ and $\alpha_{T V}$ are horizontal and vertical transverse dispersivities, respectively, $|\boldsymbol{v}|$ is the magnitude of the velocity vector, and $D_{d}$ is the effective diffusion coefficient. By using $\boldsymbol{A}$ and $\boldsymbol{B}$ as

$$
\begin{gathered}
\boldsymbol{A}=\boldsymbol{v}+\nabla \cdot \boldsymbol{D}+\frac{1}{n_{p}} \boldsymbol{D} \cdot \nabla n_{p} \\
2 \boldsymbol{D}=\boldsymbol{B} \cdot \boldsymbol{B}^{T}
\end{gathered}
$$

and noting that Eq.(4) and Eq.(5) become equivalent.

Substituting Eq.(12) and Eq.(13) into Eq. (3) yields the equation for the displacement of a particle in random walk (Uffink, 1990; Tompson, 1993; Salamon et al., 2006).

$$
\begin{aligned}
\boldsymbol{X}_{p}(t+\Delta t)=\boldsymbol{X}_{p}(t) & +\left(\boldsymbol{v}+\nabla \cdot \boldsymbol{D}+\frac{1}{n_{p}} \boldsymbol{D} \cdot \nabla n_{p}\right) \Delta t \\
& +\boldsymbol{B}\left(\boldsymbol{X}_{p}\right) \boldsymbol{\Xi}(t) \sqrt{\Delta t}
\end{aligned}
$$

The displacement matrix $\boldsymbol{B}$ has the form (Burnett and Frind, 1987b; Lichtner et al., 2002)

$$
\begin{aligned}
& \boldsymbol{B}=\left(\begin{array}{ccc}
\frac{v_{1}}{|\boldsymbol{v}|} \sqrt{2 \alpha_{L}|\boldsymbol{v}|} & \frac{-v_{1} v_{3} \sqrt{2 \alpha_{T V}|\boldsymbol{v}|}}{|\boldsymbol{v}| \Gamma} & -\frac{v_{2} \sqrt{J}}{\Gamma} \\
\frac{v_{2}}{|\boldsymbol{v}|} \sqrt{2 \alpha_{L}|\boldsymbol{v}|} & \frac{-v_{2} v_{3} \sqrt{2 \alpha_{T V}|\boldsymbol{v}|}}{|\boldsymbol{v}| \Gamma} & \frac{v_{1} \sqrt{J}}{\Gamma} \\
\frac{v_{3}}{|\boldsymbol{v}|} \sqrt{2 \alpha_{L}|\boldsymbol{v}|} & \frac{\Gamma}{|\boldsymbol{v}|} \sqrt{2 \alpha_{T V}|\boldsymbol{v}|} & 0
\end{array}\right) \\
& \Gamma=\sqrt{v_{1}^{2}+v_{2}^{2}}, \quad J=2\left(\frac{\alpha_{T H} \Gamma^{2}+\alpha_{T V} v_{3}^{2}}{|\boldsymbol{v}|}\right)
\end{aligned}
$$

Equilibrium sorption using a linear isotherm can be incorporated by replacing the velocity $\boldsymbol{v}$ with a retarded velocity $v / R$ and $D$ with $D / R$ in Eq. (14) where $R$ is the retardation factor. 
A second-order partial differential equation shown in Eq. (5) can be solved using an Eulerian approach by finite element method. However, in order to overcome the problem of artificial oscillation and numerical dispersion, the Peclet number and Courant number have to be sufficiently small. Random walk particle tracking offers free of these problems and can automatically satisfy global mass conservation due to the use of particles as discrete mass parcels.

\subsection{Temporal moments}

Temporal moments associated with observed breakthrough curves at several predefined control planes are computed using a Lagrangian framework. Monitoring the first passage time of particles passing through control planes allows for the estimation of temporal moments of breakthrough curves without having to evaluate the actual shape of the breakthrough curve. The $n$-th normalized absolute temporal moment can be calculated as the expected value of the arrival time of a particle at the control plane to the $n$-th power (Shapiro and Cvetković, 1988; Fernàndez-Garcia et al., 2005).

$$
M_{n, T}=\frac{\sum_{k=1}^{N P_{s}} m_{p}^{k}\left(t_{p}^{k}\left(x_{1}\right)\right)^{n}}{\sum_{k=1}^{N P_{s}} m_{p}^{k}}
$$

where $M_{n, T}$ is the $n$-th normalized absolute temporal moment, $x_{1}$ is the mean flow direction coordinate, $m_{p}^{k}$ is the mass assigned to the $k$-th particle, which is set constant for all particles due to the condition of no-decay, $t_{p}^{k}$ is the first arrival passage time of the $k$-th particle, $N P_{s}$ is the total number of particles arrived at the $x_{1}$-control plane. The $n$-th normalized central temporal moment is calculated using relationship between central and absolute temporal moments,

$$
\Lambda_{n, T}=\sum_{r=0}^{n}\left(\begin{array}{l}
n \\
r
\end{array}\right) M_{n-r, T}\left(x_{1}\right)\left(-M_{1, T}\left(x_{1}\right)\right)^{r}
$$

where $\Lambda_{n, T}$ is the $n$-th normalized central temporal moment.

Longitudinal macrodispersivity from temporal moments is calculated as (Goltz and Roberts, 1987)

$$
A_{11}\left(\xi_{P}\right)=\frac{\xi_{P}}{2} \frac{\Lambda_{2, T}\left(\xi_{P}\right)}{\left(M_{1, T}\left(\xi_{P}\right)\right)^{2}}
$$

where $A_{11}$ is the longitudinal macrodispersivity, $\xi_{P}$ is the distance between the source and the $x_{1}$-control plane. This is also applicable not only to conservative solutes but to reactive tracers. The advantage of particle tracking approach for computing the temporal moments as well as for identifying the longitudinal micro or macrodispersivity is that the absence of constructing the entire breakthrough curves and subsequent integration of concentrations over time (Valocchi, 1985; Inoue et al., 2007).

\subsection{Spatial moments}

One of the important aims of transport theories is to predict the extent of dilution or mixing of a body of contaminated water as it moves with the flow. A commonly used measure of dilution is the spatial moments of the concentration distribution. Spatial moments of aqueous concentrations distributed in space in a single realization are calculated from snapshots of particles at given times as follows (Kitanidis, 1988; Tompson, 1993).

$$
\begin{gathered}
m(t)=\sum_{k=1}^{N P_{t}} \frac{m_{p}^{k}}{R\left(X_{p}^{k}(t)\right)} \\
X_{G, i}=\frac{1}{m(t)} \sum_{k=1}^{N P_{t}} \frac{m_{p}^{k} X_{p, i}^{k}(t)}{R\left(X_{p}^{k}(t)\right)} \\
S_{i j}(t)=\frac{1}{m(t)} \sum_{k=1}^{N P_{t}} \frac{m_{p}^{k} X_{p, i}^{k}(t) X_{p, j}^{k}(t)}{R\left(X_{p}^{k}(t)\right)}-X_{G, i}(t) X_{G, j}(t)
\end{gathered}
$$

where $m(t)$ is the total liquid phase solute mass, $m_{p}^{k}$ is the mass assigned to the $k$-th particle, $X_{G, i}$ is the first order spatial moment and means the center of mass of particles, $S_{i j}$ is the liquid phase second spatial moments associated with the distribution of particles at a certain time, $R$ is the retardation factor at the particle location, $N P_{t}$ is the number of particles in the system at time $t$, and $X_{p, i}^{k}$ is the $i$-component of the $k$-th particle location.

Longitudinal and transverse macrodispersivities from spatial moments of the distributed particles are calculated as (Freyberg, 1986; Rajaram and Gelhar, 1993)

$$
\begin{aligned}
& A_{11}\left(\xi_{G}(t)\right)=\frac{S_{11}}{2 \xi_{G}(t)} \\
& A_{22}\left(\xi_{G}(t)\right)=\frac{S_{22}}{2 \xi_{G}(t)} \\
& A_{33}\left(\xi_{G}(t)\right)=\frac{S_{33}}{2 \xi_{G}(t)}
\end{aligned}
$$

where $A_{22}$ and $A_{33}$ are the horizontal and vertical transverse macrodispersivity, respectively, $\xi_{G}(t)$ is the travel distance of the center of mass of the plume in the mean flow direction at time $t$. This is applicable to conservative as well as reactive solutes. Dispersivities from spatial moments obtained in this work are based on particle distributions at times when none of the particles has exited the domain.

\section{Design of computational features}

\subsection{Study area and available data}

This study site is located in the dunes near The Hague in the province of Zuid Holland, the Netherlands, as shown in Figure 1. Formations consisting of a Pleistocene deposits comprise the aquifer system having a total thickness of about 19 meters. At the top and bottom the aquifer is confined by thick clay layers, providing impervious layers.

A schematic view of the field of concern is illustrated in Figure 2. The hydraulic conductivity distribution in the aquifer at the experimental site has been studied by granular analysis of soil samples at positions denoted $R_{a}$ and $R_{b}$ in Figure 2 (Uffink, 1990). The resulting profiles of hydraulic 


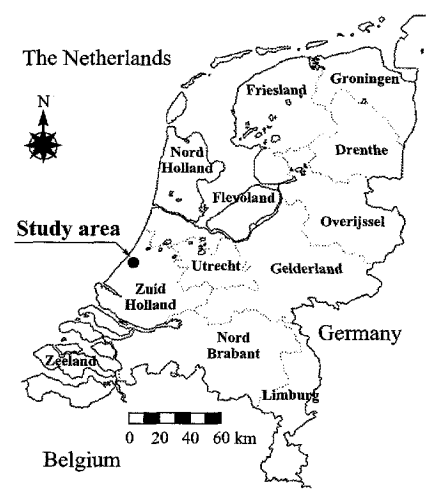

Figure 1: Location of the study area in the Netherlands.

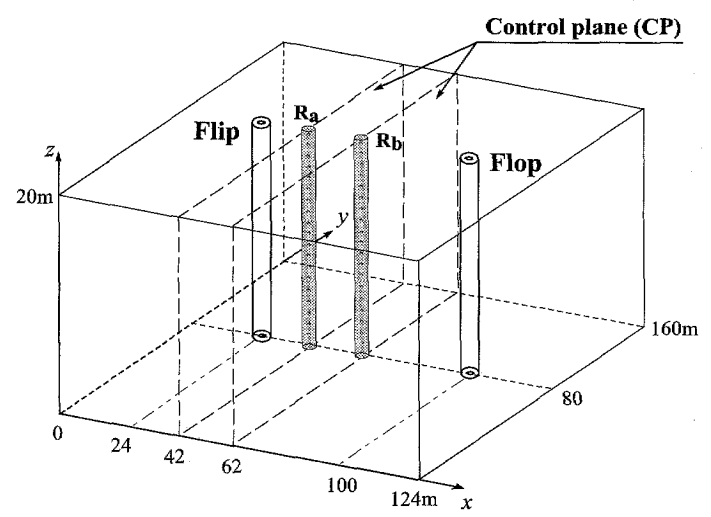

Figure 2: Sketch of the flow domain and locations of the source and the control plane.

conductivity have the arithmetic mean of hydraulic conductivity of $1.07 \mathrm{~m} /$ day and the geometrical variance $\sigma_{\ln K}^{2}$ of 0.44 and are shown in Figure 3. Uffink (1990) assumed to be the stratification of this aquifer with the porosity of 0.34 in an entire domain and conducted random walk particle tracking in a two-dimensional cross-section of aquifer where an injection well Flip, an extraction well Flop and soil sample cores $R_{a}$ and $R_{b}$ are involved.

In this study, three types of hydraulic conductivity distribution are assumed to be isotropic and are modeled to investigate the effect of spatial variability of porous formations on the macrodispersion. One of the three such possible models is chosen to treat the hydraulic conductivity distribution as "Homogeneous aquifer". Additionally, like the Uffink model, the aquifer of interest is assumed to be the stratified aquifer in an entire three-dimensional field, referred to as "Stratum aquifer". On the other hand, soil sample cores at $R_{a}$ and $R_{b}$ provide vertical variations of hydraulic conductivity as shown in Figure 3. The experimental variogram of field data from Figure 3 is presented in Figure 4 by closed circles. The solid line indicates the fitted isotropic exponential model variograms calculated using Eq. (26) (Deutsch and Journel, 1992).

$$
\gamma_{K}=n_{K}+c_{K}\left(1.0-\exp \left(\frac{-h_{K}}{\lambda_{K}}\right)\right)
$$

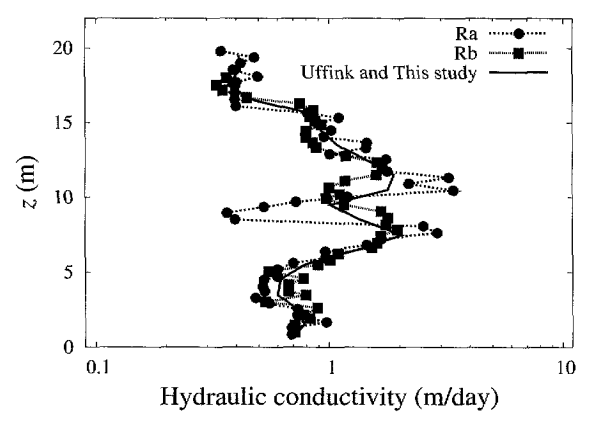

Figure 3: Hydraulic conductivity versus depth at two locations. Vertical distribution employed in this study is also depicted.

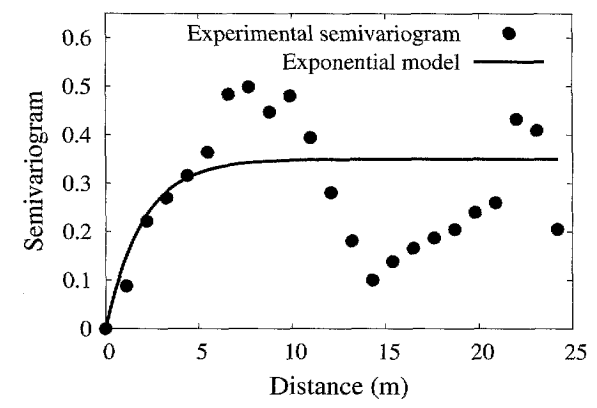

Figure 4: Experimental and fitted exponential variogram.

where $\gamma_{K}$ is the variogram, $c_{K}$ is the sill, $h_{K}$ is the lag distance, $n_{K}$ is the nugget effect and $\lambda_{K}$ is the range. Parameter values of the fitted exponential model are set to $c_{K}=0.35$, $\lambda_{K}=2.0, n_{K}=0$. As a few studies point out, the experimental variograms become noisy at larger lag distances owing to the small number of observations available (Welty and Elsner, 1997; Kobayashi et al., 1998).

In order to interpolate values of hydraulic conductivity at unsampled locations in an entire domain, ordinary kriging is used (Journel, 1989; Deutsch and Journel, 1992), providing the third possible hydraulic conductivity distribution, referred to as "Geostatistics aquifer". In geostatistics aquifer, the variation of hydraulic conductivity is limited to a narrow range around soil sample points at $R_{a}$ and $R_{b}$ due to the variogram length. All aquifer models have the same mean value of hydraulic conductivity of $1.07 \mathrm{~m} /$ day but differ in porous formations. Figure 5 illustrates a profile of hydraulic conductivity distribution in a vertical section with $y=80 \mathrm{~m}$ for Stratum model and Geostatistical model.

\subsection{Flow fields}

A steady, saturated velocity field corresponding to each hydraulic conductivity field is developed by solving the system of Eq. (1) and Eq. (2) within the three-dimensional domain where prismatic grid spacing $\Delta x=1 \mathrm{~m}$ to $10 \mathrm{~m}, \Delta y=1 \mathrm{~m}$ to $10 \mathrm{~m}$ and $\Delta z=0.5 \mathrm{~m}$ is adopted in each direction. The local head $h(\boldsymbol{x})$, i.e., the boundary condition of the first type, is specified on two sides faces intersecting the plane with $x=0$ 


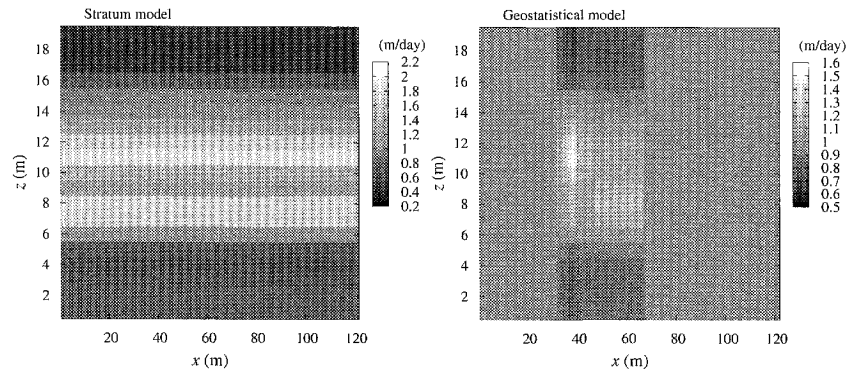

Figure 5: Hydraulic conductivity distributions: (left) Stratum model and (right) Geostatistical model. These graphs show the $x-z$ cross-section with $y=80 \mathrm{~m}$.

and $x=124 \mathrm{~m}$, while all other faces are treated as no-flow boundaries. This results in the establishment of a mean hydraulic gradient of 0.36 to reflect the pumping test reported in the literature (Uffink, 1990). As for solving the seepage equation, inflow is set to the positive in the finite element method.

Within each hydraulic conductivity distribution, three types of flow patterns are considered under the same boundary conditions for six faces. Injection and extraction wells are under the operation with a certain injection or pumping rate in the domain, and are located at Flip and Flop denoted in Figure 2, respectively. As an assumption, the total injection or pumping rate specified is divided evenly among the finite element nodes intersecting the well screen. As the base case, injection and extraction rates are set approximately to $Q(\boldsymbol{x})=480 \mathrm{~m}^{3} /$ day at Flip and $Q(\boldsymbol{x})=-480 \mathrm{~m}^{3} /$ day at Flop, respectively, in order to compare the difference of macrodispersivity outcomes between estimates of this study and data reported in the literature (Uffink, 1990). Therefore, flow fields exhibit the "doublet" or convergent flow (Huyakorn et al., 1986) in the vicinity of an injection and pumping well pair, despite of the porous formations. Moreover, two different operation conditions of both wells at onehalf and one-tenth the total rate are considered to investigate the effect of the total rate of pumping or injection on the macrodispersivity estimates. All other specifications including the boundary conditions remain unchanged from the base case.

\subsection{Particle tracking implementation}

To implement any particle tracking algorithm, a method is needed for computing the velocity vector at all locations in the model domain in order to calculate the deterministic portion of the particle transport vector, given by $\boldsymbol{A}$ in Eq. (12). In addition, application of the random walk particle tracking requires knowledge of the velocity gradient at particle location. However, when a numerical flow model such as finite element model is used to solve for the head distribution, groundwater velocity is known only at certain locations. Therefore, in this study, velocity interpolation technique (Cordes and Kinzelbach, 1992; Zheng and Bennett, 1995) is employed to compute the components of flow velocity associated with an atrbitrary location of a particle. While a finite element model
Table 1: Parameters used in this study.

\begin{tabular}{lr} 
Longitudinal dispersivity $(\mathrm{m})$ & 0.01 \\
Horizontal transverse dispersivity $(\mathrm{m})$ & 0.001 \\
Vertical transverse dispersivity $(\mathrm{m})$ & 0.001 \\
Effective diffusion coefficient $\left(\mathrm{m}^{2} /\right.$ day $)$ & 0 \\
Retardation factor & 1 \\
Porosity & 0.34 \\
Number of particles & $2 \times 10^{5}$ \\
Time increment $($ day $)$ & 1 \\
Injection rate at well $\left(\mathrm{m}^{3} /\right.$ day $)$ & $48,240,480$ \\
Pumping rate at well $\left(\mathrm{m}^{3} /\right.$ day $)$ & $48,240,480$ \\
\hline
\end{tabular}

is the computational framework on which particle tracking methods are based, random walk particle tracking method stated above is performed under the prescribed flow conditions.

As an initial pulse input, the number of particles used to represent the solute is $2 \times 10^{5}$ with a constant mass of $76800 \mathrm{~g} / 2 \times 10^{5}$ per one particle in order to reflect the mean concentration of pumped water at this site (Uffink, 1990). All particles are applied uniformly distributed at random within the injection well, Flip, as shown in Figure 2. Additionally, longitudinal dispersivity and horizontal and vertical transverse dispersivities are chosen to be $\alpha_{L}=0.01 \mathrm{~m}$, $\alpha_{T H}=0.001 \mathrm{~m}$ and $\alpha_{T V}=0.001 \mathrm{~m}$, respectively, which are based on the data reported in the literature (Uffink, 1990). When a particle arrives at a withdrawal well Flop, that particle is regarded to flow into the well and is removed from the flow domain. To simplify the problem, a particle is assumed to be conservative and not to have the adsorptive nature, leading to 1 of retardation factor. Parameters used in this analysis is listed in Table 1.

Furthermore, application of a random walk particle tracking requires that some computational approximations be established. The time step used to move a particle through space is required to be small enough because use of too large a time step may lead to overshoot errors in the displacement expressed in Eq. (14) (Tompson et al., 1995). Thus, whereas all particles move from one time level to another with an identical time step value and are allowed to transfer and disperse until a maximum duration of 1000 days, step doubling procedure (Zheng et al., 1991) is used to assure the accuracy of particle tracking linked with the fourth-order Runge-Kutta method.

Swarm of particles is advected and dispersed according to the velocity distribution toward a $(y, z)$-plane located at some distance from the injection location. This study focuses on the passage of particles through a $(y, z)$-plane, referred to as the control plane, through which the solute mass flux is to be predicted or measured. For a quantitative description of the transport and macrodispersion of particles, the breakthrough of the solute plume across the entire control plane is assessed. Several control planes are located from $x=28 \mathrm{~m}$ to $x=99 \mathrm{~m}$ in the $x$-direction to evaluate the particle behavior passing through these control planes, as seen in Figure 2. Break- 


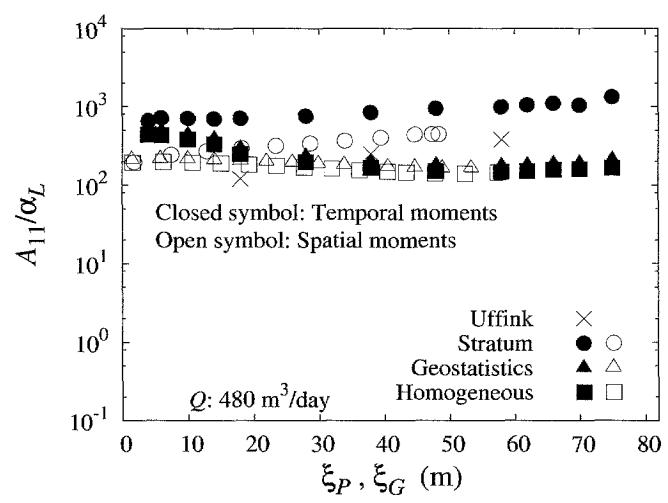

Figure 6: Comparison of longitudinal macrodispersivity estimated from temporal moments of breakthrough curves and spatial moments of tracer plume under the pumping rates of $480 \mathrm{~m}^{3} /$ day.

through curves obtained at each control plane allow to estimate the longitudinal macrodispersivity based on Eq. (19).

On the other hand, the overall development of a solute plume is able to be measured in terms of spatial moments of its mass distribution using Eq. (20) through Eq. (22). Contrary to the macrodispersivity estimated from temporal moments, spatial moment analysis is a useful tool to identify not only the longitudinal macrodispersivity but the transverse macrodispersivities using Eq. (23) and Eqs. (24) and (25), respectively. This point is one advantage of a particle tracking approach to effectively achieve the estimate of macrodispersivity in a heterogeneous flow field instead of the use of conventional finite element or finite difference techniques.

\section{Results and discussion}

\subsection{Influence of the hydraulic conductivity distribution}

Longitudinal macrodispersivity estimates from temporal and spatial moments normalized by the local or microdispersivity as a function of the travel distance along $x$-direction from the injection well is illustrated in Figure 6 under the absolute pumping/injection condition of $480 \mathrm{~m}^{3} /$ day. Reported estimates (Uffink, 1990) at three points are also plotted in Figure 6. Although all simulations run for a period of time long enough to construct a relatively complete downstream breakthrough curve, spreading and displacement characteristics in terms of spatial moments of the particle distribution may be limited to a certain time period because all particles are required to exist within the domain to ensure the accuracy of statistical moments. According to the porous formation and the activity of well, an arrival time of particle to the pumping well varies significantly, leading to the difference of macrodispersivity evolution estimated from spatial moments.

As shown in Figure 6, macrodispersivity estimates range from $10^{2}$ to $10^{3}$ and are almost identical to the results obtained by Uffink, despite of the hydraulic conductivity distribution. Whereas both temporal and spatial moments provide the low degree of macrodispersivity increase as well as the results of Uffink in stratum aquifer, while the change

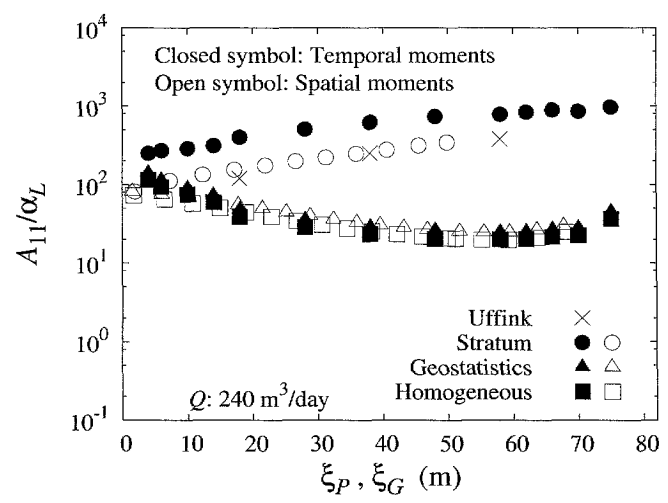

Figure 7: Effect of the degree of extraction rate at the pumping well on the longitudinal macrodispersivity under the pumping rates of $240 \mathrm{~m}^{3} /$ day.

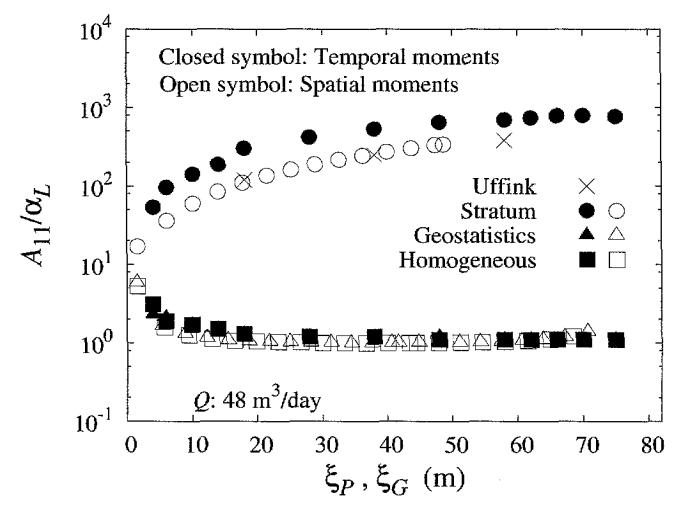

Figure 8: Effect of the degree of extraction rate at the pumping well on the longitudinal macrodispersivity under the pumping rates of $48 \mathrm{~m}^{3} /$ day.

of macrodispersivities both for geostatistics and homogeneous aquifers remains approximately constant. Generally, whereas typical values of local dispersivity in laboratory experiments range between 0.001 and $0.1 \mathrm{~m}$ (e.g., $\mathrm{Xu}$ and Eckstein, 1997; Guedes de Carvalho and Delgado, 2003; Inoue et al., 2006), values of macroscopic dispersivity are two to four orders of magnitude larger (e.g., Neuman, 1990; Gelhar et al., 1992). This disparity arises because dispersion at the field scale is caused primarily by macroscopic heterogeneities, rather than by pore-scale heterogeneity. Fieldscale dispersivity increases with distance and with time and is influenced to the stratification of an aquifer (Gelhar and Axness, 1983, Uffink, 1983). Consequently, Figure 6 demonstrates that evolution of macrodispersivities from both temporal and spatial moments in stratum aquifer is appropriate.

In order to investigate the effect of the degree of pumping rate at withdrawal well, macrodispersivity estimates for all hydraulic conductivity distributions as a function of the travel distance under the pumping rates of $240 \mathrm{~m}^{3} /$ day and $48 \mathrm{~m}^{3} /$ day are plotted in Figures 7 and 8, respectively. When a pulse of solute mass introduces into a flow field behavior of macrodispersivity is erratic with time, particularly very close 

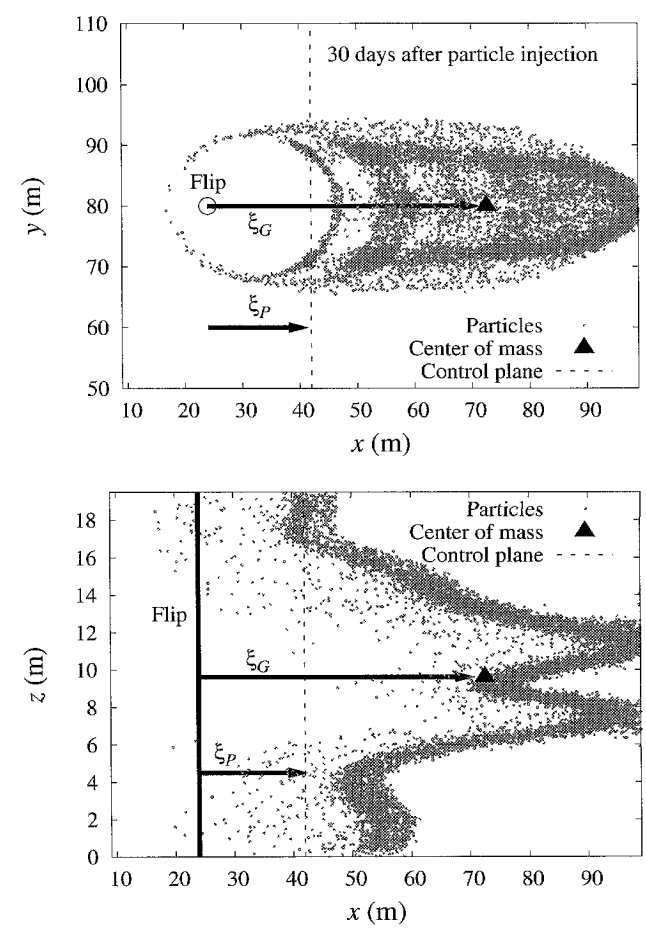

Figure 9: Disparity between macrodispersivities estimated from temporal and spatial moments: (top) plan view of the particle cloud illustrating the location of the control plane and (bottom) vertical view of the particle cloud with the center of mass.

to a source (Molz et al., 1983; Tompson and Gelhar, 1990). As the plume continues to move, it will expand and gradually be characterized after a certain displacement occurs. In stratum aquifer, macrodispersivities from temporal moments approach an asymptotic value, while macrodispersivities from spatial moments increase with the increase of travel distance and are in good agreement with the results of Uffink (1990). It can be observed that stratum aquifer exhibits less sensitive to flow paths when computing the macrodispersivity. Thus, in layered porous formations macrodispersivity can be estimated using either temporal or spatial moments despite of the pumping rate of the injection and extraction wells.

On the other hand, variation of macrodispersivities in geostatistics and homogeneous aquifers is substantially sensitive to the change of pumping rates. As for the case with $48 \mathrm{~m}^{3} /$ day of pumping rate, values of macrodispersivities are approximately 1.0 , except for a range within a relatively short travel distance, indicating that macrodispersivity is identical to microdispersivity. In geostatistics aquifer of interest, behavior of particles appears to be similar to than in homogeneous aquifer due to the less heterogeneity of aquifer with 0.44 of geometric variance as shown in Figure 5. Therefore, the structure of hydraulic conductivity distribution in an entire domain in geostatistics aquifer may be regarded as a homogeneous formation, although the hydraulic conductivity varies partially in the vicinity of sampling positions $R_{a}$ and $\mathrm{R}_{\mathrm{b}}$. Moreover, as pointed out by Goltz and Roberts (1987) and Inoue et al. (2007), both temporal and spatial moments

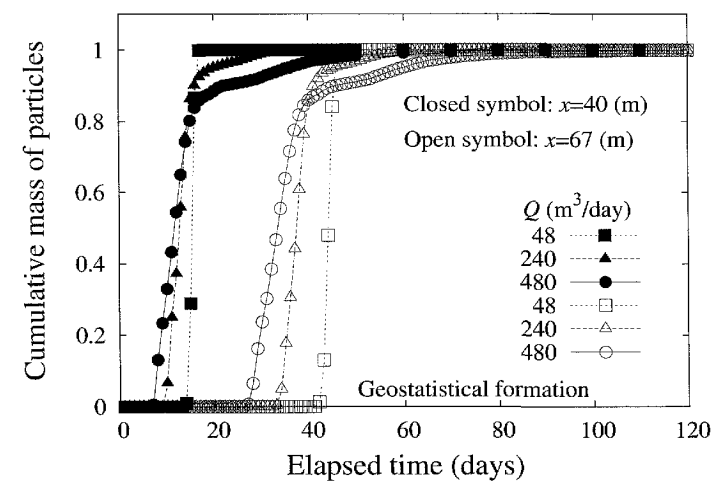

Figure 10: Variation of cumulative mass of particles at control planes in geostatistics aquifer.

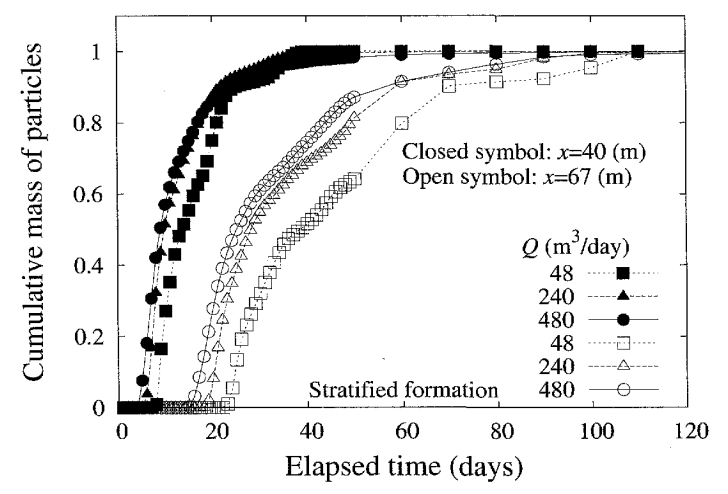

Figure 11: Variation of cumulative mass of particles at control planes in stratum aquifer.

are capable of providing the microdispersivity in homogeneous porous formation under a uniform flow condition. The pumping rate of $48 \mathrm{~m}^{3} /$ day is approximately $3 \%$ of the rate of flow in volume of water of the domain and appears to be relatively small. Hence, when a convergent, or non-uniform flow may be treated as a uniform flow in geostatistics and homogeneous aquifers of concern under the low pumping rate condition such as $48 \mathrm{~m}^{3}$ /day in this study, temporal and spatial moments are capable of assessing the microdispersivity, instead of the macrodispersivity.

\subsection{Disparity of macrodispersivity}

As seen in Figures 6 through 8, in stratum aquifer, macrodispersivities estimated from spatial versus temporal moments exhibit different scale-dependent behavior, while the influence of pumping rate on the asymptotic values estimated from spatial and temporal moments is not significant. As pointed out by Dagan (1984) and Naff (1990), the asymptotic constant values are expected to be almost the same for both moments of macrodispersivities as travel distance increases. However, there are differences in the distance-scale at which the macrodispersivities achieve a constant value in aquifer of concern. It is evident from Figures 6 through 8 that the distance-scale at which macrodispersivities from temporal moments of breakthrough curves at a control plane ap- 


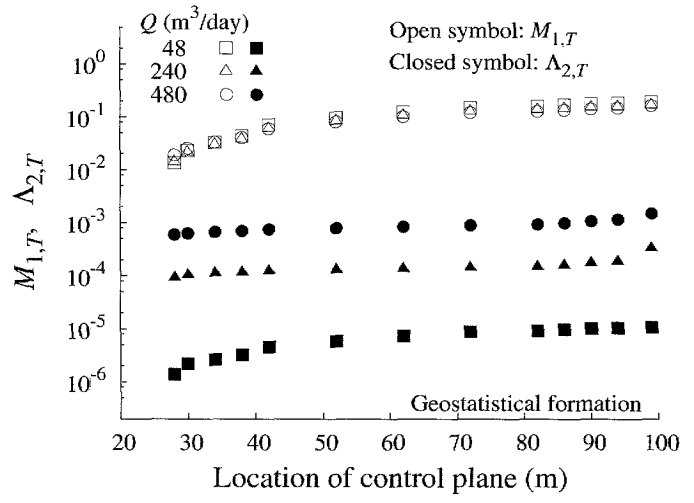

Figure 12: Variation of the first absolute and second central temporal moments as a function of location of control planes in geostatistics aquifer.

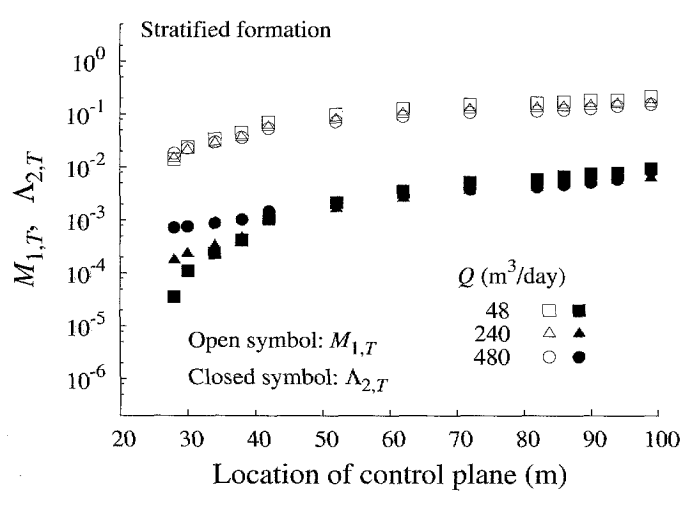

Figure 13: Variation of the first absolute and second central temporal moments as a function of location of control planes in stratum aquifer.

proach an asymptotic value is smaller that the corresponding distance-scale for macrodispersivities estimated from spatial moments of particle cloud. The degree of this disparity between macrodispersivities estimated from temporal and spatial moments is in common regardless of a prescribed pumping rate.

Figure 9 illustrates the particle cloud of a tracer in plan and vertical views as one example to explain the behavior for stratum aquifer under the pumping condition of $240 \mathrm{~m}^{3} /$ day. To simplify a snapshot of particle distribution, a swarm of $10^{4}$ particles 30 days after injection and its centroid in $x, y$ and $z$ coordinates are plotted. A breakthrough curve assessed at a control plane described in Figure 9 provides almost the same macrodispersivity that obtained from the second spatial moment corresponding to a specific plume centroid location, also shown in the figure. All particles applied at the source, i.e., Flip in this study, are required to pass through a specific control plane to express a complete breakthrough curve and to lead macrodispersivity using temporal moments. On the contrary, application of spatial moment method is restricted since all particles emerged at the injection point are necessary to identify the estimates. Whereas spatial moment method is capable of leading a value as macrodispersivity at a corresponding travel time as long as all particles exist in the aquifer, larger times are necessary to assess an appropriate macrodispersivity based on temporal moment method. If at larger times as mentioned above, both temporal and spatial moments could provide the same asymptotic macrodispersivity despite of the flow condtion, as reported by Dagan (1986) and Naff (1990). Hence, in flow fields in this study, due to a restriction of travel distance of particles, macrodispersivity estimates from temporal moments become larger those from spatial moments.

\subsection{Characteristics of temporal and spatial moments}

The relative cumulations of mass breakthrough curves divided by the total mass of application at the source in geostatistics aquifer are shown in Figure 10 as for representative two control planes for all pumping rate conditions. It can be observed that the number of particles passing through a control plane varies abruptly, especially for the case with the smallest pumping rate. A time series mass variation indicates that breakthrough curves exhibit a similar Gaussian shape regardless of the location of a control plane, although a slight tailing of breakthrough curves can be seen for the largest pumping rate. This point attributes to the similarity of geostatistics aquifer of interest to the homogeneous aquifer where the evolution of cumulative mass of particles shows a Gaussian distribution (Welty and Gelhar, 1994; Inoue et al., 2007).

In contrast to the results depicted in Figure 10, variation of cumulative mass of particles at two control planes in stratum aquifer is shown in Figure 11. It is apparent from the plot that the number of particles arriving at a control plane gradually increases and varies in a stepwise fashion after approximately half the number of particles pass through the control planes. This plume behavior reflects the hydraulic conductivity distribution on which the evolution of plume is considerably dependent.

As stated above, alike from Eulerian approach such as finite element or finite difference method, construction of the entire breakthrough curves and subsequent integration of mass variation with respect to time are not required to evaluate temporal moments in particle tracking approach. Temporal moments associated with each control plane are calculated and utilized to determine the longitudinal macrodispersivity (Valocchi, 1985; Goltz and Roberts, 1988). To quantify the values of temporal moments, the first absolute temporal moments $M_{1, T}$ in Eq. (17) and the second central temporal moments $\Lambda_{2, T}$ in Eq. (18) in geostatistics and stratum aquifers are plotted in Figures 12 and 13, respectively.

The most noticeable quantitative difference is relevant to the variation of the second central temporal moments denoted by closed symbols in these figures. While the second central temporal moments $\Lambda_{2, T}$ in geostatistics aquifer vary corresponding to the pumping rate as well as the location of control plane, it is interesting to note that stratum aquifer provides an asymptotic value of the second central temporal moments regardless of the pumping rate, except for a location relatively closer to the source. It is inferred that as for 

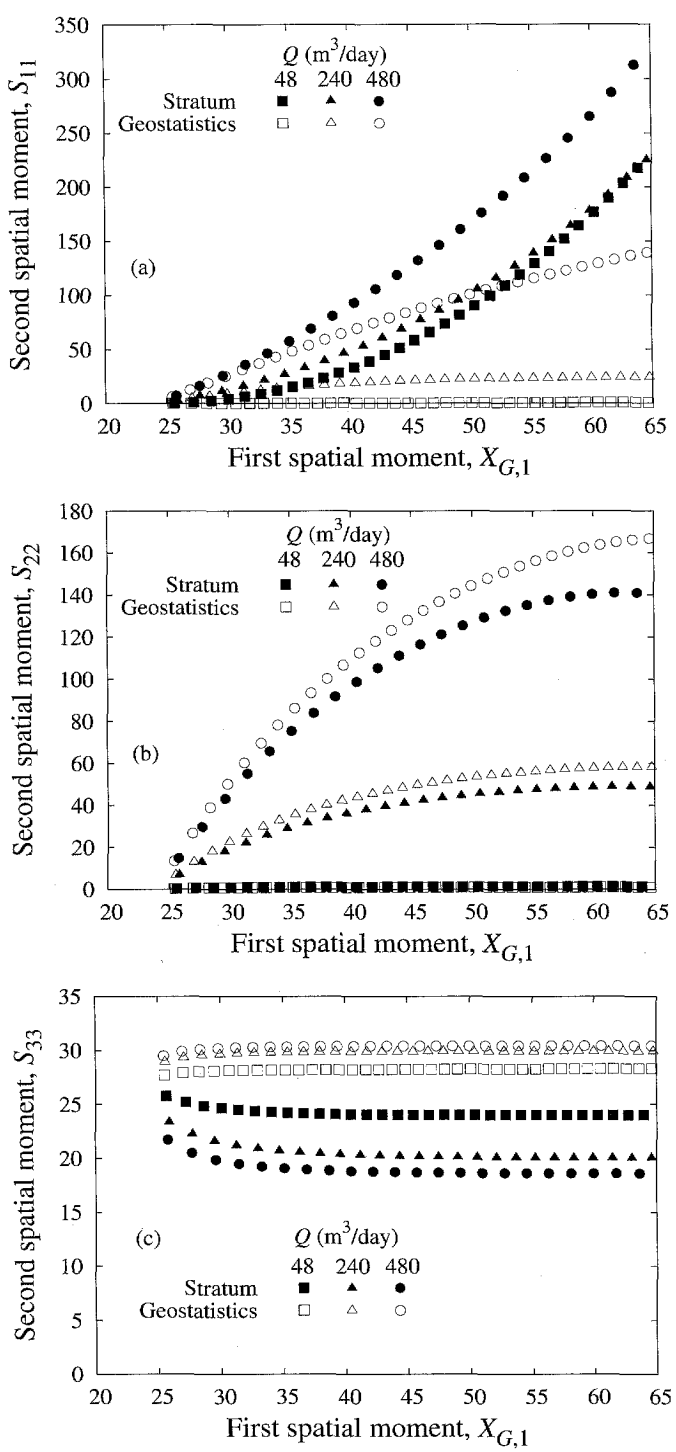

Figure 14: Evolution of the second spatial moments as a function of the first spatial moments under different pumping conditions: (a) $S_{11}$, (b) $S_{22}$ and (c) $S_{33}$.

stratum aquifer, the effect of hydraulic conductivity distribution on plume evolution is much more pronounced compared to the effect of pumping rate because plume behavior in aquifer depends highly not only on the hydraulic conductivity of a layer at which a particle is located but also on the vertical mixing of particles between layers (Uffink, 1983; Hoteit et al., 2002). Hence, eventually, the marked difference of asymptotic values between geostatistics and stratum aquifers can be observed in Figures 6 and 7 .

In order to characterize the evolution of particle swarm in each direction, the principal components of the tracer plume spreading variance tensor $S_{i i}$ are plotted as a function of the first spatial moment $X_{G, 1}$ in $x$-coordinate for all pumping rate conditions in both geostatistics and stratum aquifers in Figure 14. These components can be used to gauge the size of a particle cloud in a given direction and determine the rate

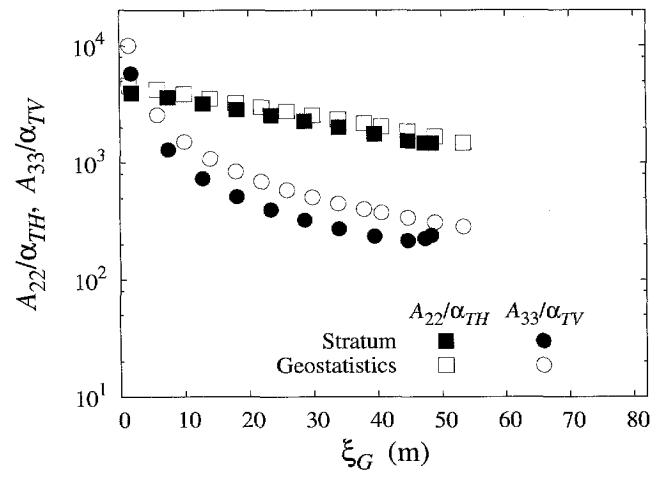

Figure 15: Comparison of horizontal and vertical transverse macrodispersivities estimated from spatial moments under the pumping rate of $480 \mathrm{~m}^{3} /$ day in geostatistics and stratum aquifers.

of growth as a function of mean displacement. They may be used to assess the nature of macrodispersion, and in particular, to determine whether constant macrodispersivity components can be identified and measured.

The predominant axis of spreading and the nonlinearity of the growth occur in the longitudinal direction for stratum aquifer (Figure 14(a)). Transverse spread variance perpendicular to the longitudinal axis shown in Figure 14(b) has its own asymptotic value, while that in the vertical direction (Figure 14(c)) exhibits still another behavior. This means that velocity distribution and porous formation in space contribute to the elongation of particles in each direction. As a whole, each set of curves indicates a marked degree of variability corresponding to the flow condition. Increase or decrease of the second order spatial moments may reflect the stretching and squeezing each particle cloud undergoes during displacement. These different growth may be due to a developmental process (Dagan, 1984) associated with generated plume expansion, variabilities associated with the plume moving through different hydrogeologic layers, or a combination of both (Tompson et al., 1995).

The transverse macrodispersivity components $A_{22}$ and $A_{33}$ estimated from spatial moments using Eq. (24) and Eq. (25) and normalized by local dispersivities of $\alpha_{T H}$ and $\alpha_{T V}$, respectively, are plotted as a function of displacement distance in Figure 15. Since the variation of the transverse macrodispersivities does not depend upon the pumping rate, estimates under the condition of pumping rate of $480 \mathrm{~m}^{3} /$ day are shown as a representative outcome. Both horizontal and vertical transverse dispersivities decrease with the increase of travel distance of particle cloud and eventually reach more than three and two order of magnitude larger than local horizontal and vertical transverse dispersivities, respectively. Dagan (1986) and Rajaram and Gelhar (1993) suggest the asymptotic value of the transverse macrodispersivities is zero under uniform flow condition. It is interesting that under non-uniform flow condition nonzero values of $A_{22}$ and $A_{33}$ are obtained as far as travel distance of particles is bounded and is in the range of approximately $100 \mathrm{~m}$. Nevertheless, 
contrary to the use of temporal moments, it is emphasized that spatial moments allow to estimate not only the longitudinal macrodispersivity but the transverse macrodispersivity. In the assessment of the fate and transport of contaminants in a field, as hydraulic properties of subsurface materials cannot by fully characterized in a deterministic way, stochastic methods and modeling of the data must be invoked. Stochastic analysis such as random walk particle tracking provides the reliable macrodispersion relevant to the variability and the spatial structure associated with hydraulic properties of the heterogeneous medium under different flow conditions.

\section{Conclusions}

Disparity of macrodispersivities estimated from temporal moments of mass breakthrough curves and spatial moments of particle plumes has been investigated through random walk particle tracking approach in homogeneous and heterogeneous porous formations, which reflect the soil samples in the field in the southwest of the Netherlands. The variation of macrodispersivity under non-uniform, or convergent flow conditions linked with the activity of injection and withdrawal wells has been focused on.

For stratum model, as the travel distance increases, longitudinal macrodispersivities from temporal and spatial moments increase and are in good agreement with the results of Uffink (1990). Dispersive processes occurring in the aquifer due to heterogeneity are captured differently in breakthrough curves at control planes than in snapshots of particle cloud. When macrodispersivities are assessed based on observed breakthrough curves, all particle generated in the aquifer are required to pass through a given control plane, leading to the disparity of macrodispersivities between temporal and spatial moments.

On the other hand, temporal and spatial moments in homogeneous and geostatistics aquifers of concern based on the soil samples in the field provide almost the same variation of macrodispersivities due to less heterogeneity of the aquifer. Additionally, it is revealed that stratum aquifer is less sensitive to flow paths when computing the macrodispersivity, although the values of macrodispersivity in homogeneous and geostatistics aquifers depend highly on the pumping rates not on the structure of porous formations.

The variation of the temporal moments as well as the spatial moments has been evaluated as quantitative measures of moving particles in the system. The evolution of particle cloud is substantially affected by the hydraulic conductivity distribution. It is worth noting that the second central temporal moments in stratum aquifer approach an asymptotic value despite of the pumping rate at larger travel distance, while the degree of growth with regard to the second spatial moments varies considerably under a corresponding condition, reflecting the effect of velocity distribution and porous formation in space on the elongation of particles in each direction. Moreover, the spatial moments of particle plumes serve as a tool for estimating not only the longitudinal macrodispersivity but the transverse dispersivity. This point indicates the possibility of their application to field tests in the context of predicting the behavior of large contaminant plumes.

\section{References}

[1] Bear, J. (1972): Dynamics of fluids in porous media, Dover Publications, 764p.

[2] Burnett, R.D. and Frind, E.O. (1987a): Simulation of contaminant transport in three dimensions, 1 . The alternating direction Galerkin technique, Water Resour. Res., 23(2), pp.683-694.

[3] Burnett, R.D. and Frind, E.O. (1987b): Simulation of contaminant transport in three dimensions, 2. Dimensionality effects, Water Resour. Res., 23(2), pp.695-705.

[4] Cordes, C. and Kinzelbach, W. (1992): Continuous groundwater velocity fields and path lines in linear, bilinear, and trilinear finite elements, Water Resour. Res., 28(11), pp.29032911.

[5] Guedes de Carvalho, J.R.F. and Delgado, J.M.P.Q. (2003): The effect of fluid properties on dispersion in flow through packed beds, AIChE Journal, 49, pp.1980-1985.

[6] Dagan, G. (1984): Solute transport in heterogeneous porous formations, J. Fluid Mech., 145, pp.151-177.

[7] Deutsch, C.V. and Journel, A.G. (1992): GSLIB: Geostatistical software library and user's guide, Oxford University Press, 340p.

[8] Fernàndez-Garcia, D., Illangasekare, T.H. and Rajaram, H. (2005): Differences in the scale-dependence of dispersivity estimated from temporal and spatial moments in chemically and physically heterogeneous porous media, Adv. Water Resour., 28, pp.745-759.

[9] Freyberg, D.L. (1986): A natural gradient experiment on solute transport in a sand aquifer 2. Spatial moments and the advection and dispersion of nonreactive tracers, Water Resour. Res., 22(13), pp.2031-2046.

[10] Gardiner, C.W. (2004): Handbook of Stochastic Methods: For Physics, Chemistry and the Natural Sciences, The Third Edition, Springer-Verlag, $415 \mathrm{p}$.

[11] Gelhar, L.W. and Axness, C.L. (1983): Three-dimensional stochastic analysis of macrodispersion in aquifers, Water Resour. Res., 19(1), pp.161-180.

[12] Gelhar L.W., Welty, C. and Rehfeldt, K.W. (1992): A critical review of data on field-scale dispersion in aquifers, Water Resour. Res., 28(7), pp.1955-1974.

[13] Goltz, M.N. and Roberts, P.V. (1987): Using the method of moments to analyze three-dimensional diffusion-limited solute transport from temporal and spatial perspectives, Water Resour. Res., 23(8), pp.1575-1585.

[14] Hoteit, H., Mose, R., Younes, A., Lehmann, F. and Ackerer, $\mathrm{Ph}$. (2002): Three-dimensional modeling of mass transfer in porous media using the mixed hybrid finite elements and the random-walk methods, Math. Geol., 34(4), pp.435-456.

[15] Huyakorn, P.S., Andersen, P.F., Güven, O. and Molz, F.J. (1986): A curvilinear finite-element model for simulating two-well tracer tests and transport in stratified aquifers, $\mathrm{Wa}$ ter Resour. Res., 22(5), pp.663-678.

[16] Inoue, K., Kobayashi, A., Matsunaga, N. and Tanaka, T. (2007): Application of particle tracking method to dispersivity identification and its experimental verification, J. Rainwater Catchment Systems, 13(2), pp.7-16.

[17] Inoue, K., Masaki, I. and Tanaka, T. (2006): Parameter estimation and contaminant source characterization in horizontal tracer experiment in two-layered porous media, J. Rainwater Catchment Systems, 12(1), pp.1-9.

[18] Itô, K. (1951): On stochastic differential equations, Mem. Amer. Math. Soc., 4, pp.1-51. 
[19] Journel, A.G. (1989): Fundamentals of geostatistics in five lessons, short course in Geology, American Geophysical Union, 40p.

[20] Kitanidis, P.K. (1988): Prediction by the method of moments of transport in a heterogeneous formation, J. Hydrol., 102, pp. $453-473$.

[21] Kobayashi, A., Hinata, H. and Fujii, K. (1998): Back analysis of hydraulic conductivity distribution with geostatistical simulation results as prior information, J. Appl. Mech., JSCE, 1, pp.129-136 (in Japanese).

[22] LeBlanc, D.R., Garabediel, S.P., Hess, K.M., Gelhar, L.W., Wuadri, R.D., Stollenwerk, K.G. and Wood, W.W. (1991): Large-scale natural gradient tracer test in sand and gravel, Cape Cod, Massachusetts 1: Experimental design and observed tracer movement, Water Resour. Res., 27(5), pp.895910.

[23] Lichtner, P.C., Sharad, K. and Bruce, R. (2002): New form of dispersion tensor for axisymmetric porous media with implementation in particle tracking, Water Resour. Res., 38(3), pp.21(1)-21(16).

[24] Lee, S.-Y., Carle, S.F. and Fogg, G.E. (2007): Geologic heterogeneity and a comparison of two geostatistical models: sequential Gaussian and transition probability-based geostatistical simulation, Adv. Water Resour, 30, pp.1914-1932.

[25] Mackay, D.M., Freyberg, D.L., Robberts, P.V. and Cherry, J.A. (1986): Natural gradient experiment on solute transport in a sand aquifer 1: Approach and overview of plume movement, Water Resour. Res., 22(13), pp.2017-2029.

[26] Molz, F.J., Güven, O. and Melville, J.G. (1983): An examination of scale-dependent dispersion coefficients, Ground Water, 21(6), pp.715-725.

[27] Naff, R.L. (1990): On the nature of the dispersive flux in saturated heterogeneous porous media, Water Resour. Res., 26(5), pp.1013-1026.

[28] Neuman, S.P. (1990): Universal scaling of hydraulic conductivities and dispersivities in geologic media, Water Resour. Res., 26(8), pp.1749-1758.

[29] Rajaram, H. and Gelhar, L.W. (1993): Plume scale-dependent dispersion in heterogeneous aquifers 1 . Lagrangian analysis in a stratified aquifer, Water Resour. Res., 29(9), pp.3249-3260.

[30] Salamon, P., Fernàndez-Garcia, D. and Gómez-Hernández, J. (2006): A review and numerical assessment of the random walk particle tracking method, J. Contam. Hydrol., 87, pp.277-305.
[31] Shapiro, A.M. and Cvetković, V.D. (1988): Stochastic analysis of solute arrival time in heterogeneous porous media, $\mathrm{Wa}$ ter Resour. Res., 24(10), pp.1711-1718.

[32] Taylor, S.R. and Howard, K.W.F. (1987): A field study of scale-dependent dispersion in a sandy aquifer, J. Hydrol., 90, pp.11-17.

[33] Tompson, A.F.B. (1993): Numerical simulation of chemical migration in physically and chemically heterogeneous porous media, Water Resour. Res., 29(11), pp.3709-3726.

[34] Tompson, A.F.B. and Gelhar, L.W. (1990): Numerical simulation of solute transport in three-dimensional, randomly heterogeneous porous media, Water Resour. Res., 26(10), pp.25412562.

[35] Tompson, A.F.B., McKereghan, P.F. and Nichols, E.M. (1995): Preliminary simulation of contaminant migration in ground water at the Lawrence Livermore National Laboratory, Rep. UCRL-ID-115991, Lawrence Livermore National Laboratory, $164 \mathrm{p}$.

[36] Uffink, G.J.M. (1983): A random walk method for the simulation of macrodispersion in a stratified aquifer, Relation of Groundwater Quantity and Quality, IAHS Publ., 146, pp.103114.

[37] Uffink, G.J.M. (1990): Analysis of dispersion by the random walk method, Ph.D. Dissertation, Delft University of Technology, 150p.

[38] Valocchi, A. (1985): Validity of the local equilibrium assumption for modeling sorbing solute transport through homogeneous soils, Water Resour. Res., 21(6), pp.808-820.

[39] Welth, C. and Elsner, M.M. (1997): Constructing correlated random fields in the laboratory in observations of fluid flow and mass transport, J. Hydrol., 202, pp.192-211.

[40] Welty, C. and Gelhar, L.W. (1994): Evaluation of longitudinal dispersivity from nonuniform flow tracer tests, J. Hydrol., 153, pp.71-102.

[41] Zheng, C., Bennett G.D. and Andrews, C.B. (1991): Analysis of ground water remedial alternatives at a Superfund site, Ground Water, 29(6), pp.838-848.

[42] Zheng, C. and Bennett, G.D. (1995): Applied contaminant transport modelling, theory and practice, Van Nostrand Reinhold, 440p.

[43] Xu, M. and Eckstein, Y. (1997): Statistical analysis of the relationships between dispersivity and other physical properties of porous media, Hydrogeology J., 5, pp.4-20.

Discussion open until December 31, 2010 